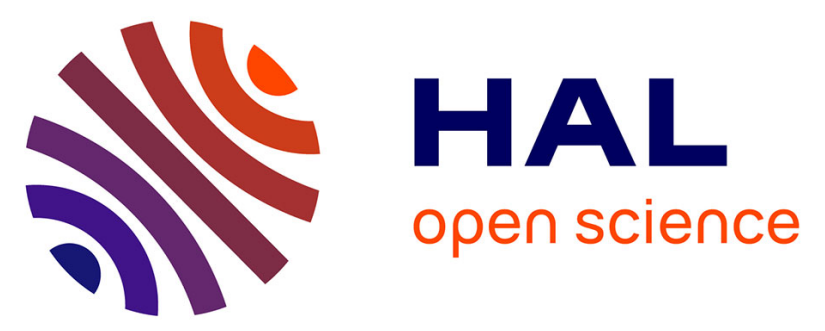

\title{
Direct comparison between in situ and VHF oblique radar measurements of refractive index spectra: A new successful attempt
}

Hubert Luce, Francis Dalaudier, Michel Crochet, Claude Sidi

\section{- To cite this version:}

Hubert Luce, Francis Dalaudier, Michel Crochet, Claude Sidi. Direct comparison between in situ and VHF oblique radar measurements of refractive index spectra: A new successful attempt. Radio Science, 1996, 31 (6), pp.1487-1500. 10.1029/96RS02087 . insu-03540211

\section{HAL Id: insu-03540211 https://hal-insu.archives-ouvertes.fr/insu-03540211}

Submitted on 24 Jan 2022

HAL is a multi-disciplinary open access archive for the deposit and dissemination of scientific research documents, whether they are published or not. The documents may come from teaching and research institutions in France or abroad, or from public or private research centers.
L'archive ouverte pluridisciplinaire $\mathbf{H A L}$, est destinée au dépôt et à la diffusion de documents scientifiques de niveau recherche, publiés ou non, émanant des établissements d'enseignement et de recherche français ou étrangers, des laboratoires publics ou privés.

$$
\text { Copyright }
$$




\title{
Direct comparison between in situ and VHF oblique radar measurements of refractive index spectra:
}

\section{A new successful attempt}

\author{
Hubert Luce, ${ }^{1}$ Francis Dalaudier, ${ }^{2}$ Michel Crochet, ${ }^{1}$ and Claude Sidi ${ }^{2}$
}

\begin{abstract}
A direct comparison between in situ and radar measurements of temperature spectra of atmospheric turbulence was performed by Dalaudier et al. [1989]. The widely accepted hypothesis of scattering from isotropic turbulence in the inertial subrange was used for the oblique reconstructions. However, the results were "puzzling" because neither the shape, the dynamic range, nor the power level was correctly reproduced. Other investigations were thus needed in order to determine the causes of these disagreements. A new attempt was performed using essentially the same method based on the same model and a more extensive and improved data set. The results, presented here, are now very satisfying for most of measurements. This agreement allows us to understand that some errors and technical problems were at the origin of the discrepancies described by Dalaudier et al. [1989]. From the new results it can be concluded that the method used, based on the isotropic model in the inertial subrange, is adequate to fairly reproduce most of the VHF radar measurements at $15^{\circ}$ from the zenith.
\end{abstract}

\section{Introduction}

It is now well-known that stratospheric-tropospheric (ST) VHF radar echoes are produced by refractive index fluctuations in air. In particular, Luce et al. [1995] showed that partial reflection from temperature sheets, described by Dalaudier et al. [1994a], is the main mechanism contributing to vertical echo enhancements. The mechanism generally proposed (and accepted) to explain the backscattered power from zenith angles $\alpha$ greater than $10^{\circ}$ was the scattering from atmospheric turbulence in the isotropic inertial subrange. Indeed, the isotropic origin of the backscattering process (for $\alpha>10^{\circ}$ ) is observed using an azimuthal beam-swinging method [e.g., Rüster et al., 1980; Röttger et al., 1981, Tsuda et al., 1986, Hocking et al., 1990]. A theoretical discussion of the isotropic and inertial range hypothesis at VHF radar sensitivity scales within turbulent layers

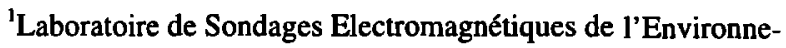
ment Terrestre, Université de Toulon et du Var,CNRS, La Garde, France.

${ }^{2}$ Service d'Aéronomie du CNRS, Verrières Le Buisson, France.

Copyright 1996 by the American Geophysical Union

Paper number 96RS02087.

0048-6604/96/96RS-02087\$11.00
}

may also be found [Hocking, 1985]. When the refractive index inhomogeneities contributing to the oblique VHF echoes (with a scale corresponding to half the radar wavelength) are within an inertial subrange, the oblique received power can be expressed in terms of the refractive index turbulent structure constant $C_{n}^{2}$ [e.g., Tatarski, 1961; Ottersten, 1969b]. This parameter describes the variability of the refractive index $n$ within the isotropic inertial subrange.

Assuming this hypothesis, an attempt for a direct comparison between VHF radar and balloon measurements was performed by Dalaudier et al. [1989]. The method relied upon nearly colocated in situ temperature measurements in order to estimate the three-dimensional (3D) spectra of refractive index fluctuations assumed to be measured by radar at the Bragg wavelength. The results of this comparison were "puzzling" (as mentioned in the title of their paper) because neither the shape, the dynamic range, nor the power level was correctly reproduced. The causes of the discrepancies are now well understood and are to be found in a combination of errors and technical problems: on the side of radar measurements, the power calibration was inaccurate; on the side of the in situ measurements, spectral levels were strongly over corrected because of errors in the estimated time constant of the thermistors. Furthermore, random contaminations on temperature measurements by tur- 
bulent wakes from the balloon or the instrumentation itself, reducing the dynamic range and the quality of the shape reconstruction, were also identified recently.

A new attempt of direct comparison was among the aims of the RASCIBA90 (radars, scidar, balloons) campaign. Both improved instrumentation and data processing allowed us to obtain satisfying results, which are the main content of this paper.

This paper is organized as follows: In section 2, general characteristics of atmospheric turbulence deduced from radar and in situ measurements will be briefly reviewed. In section 3 , some previous approaches showing experimental evidence of links between the turbulence field and radar echo intensity will first be presented. Then, Dalaudier et al.'s [1989] method will be introduced and the detailed reasons of the disagreements leading to the "puzzling result" for oblique reconstructions will be given.

In section 4 the experimental setup of the RASCIBA90 campaign will be described. Four simultaneous balloon and radar data sets are available. For each instrument the methods used in order to deduce the relevant model parameters will be briefly recalled.

In section 5 the data processing procedures applied to balloon measurements for the direct comparisons will be presented. In particular, the practical method used to estimate the 3D spectrum of refractive index from temperature measurements and the method used in order to take into account the humidity will be described. All the technical and data processing improvements with respect to Dalaudier et al. [1989] will then be reviewed. In section 6 the comparisons between radar observations and reconstructions will be presented. Three of the four data sets, corresponding to three different nights of measurements, allow consistent reconstructions of the radar echo profiles; they will be discussed first. The fourth experiment shows significant discrepancies between reconstructed and observed profiles, within some altitude range. These discrepancies will be discussed and explained by instrumental wake contamination of the in situ measurements.

In section 7, conclusions and perspectives will be finally presented.

\section{Characteristics of Atmospheric Turbulence}

From the beginning of aircraft flights the distribution of atmospheric clear air turbulence (CAT) was observed to be highly inhomogeneous because the turbulence grows and decays in layers or "patches." This characteristic was confirmed, for example, by Barat [1982] with in situ (balloon) measurements. Schematically, this patchiness appears on balloon measurements as a vertical succession of turbulent layers and quiet regions and could be at the origin of enhancements or gaps on UHF radar power profiles [e.g., Sato and Woodman, 1982]. These structures are present at all altitudes: turbulent layers were observed by VHF radars in the troposphere, the stratosphere, and the mesosphere by Woodman and Guillén [1974].

From several kinds of in situ studies (balloon, aircraft, etc) and radar measurements the turbulent layers seem to be rather thin but often largely extended in a nearly horizontal direction. From in situ measurements their thickness was estimated to be generally of the order of a few hundreds of meters and even of a few tens of meters for several of them [see Crane, 1980, Figure 1 and references herein]. With a $430-\mathrm{MHz}$ radar, Sato and Woodman [1982] identified layers of about $50 \mathrm{~m}$ thickness. With the same radar, Woodman and Rastogi [1984] observed layers less than 100-500 m thick. lerkic et al. [1990], with the Arecibo radar $(2380 \mathrm{MHz}$ and 20 -m resolution), observed layers from 60 to $300 \mathrm{~m}$ thick with 100- to 800-m spacing. Palmer et al. [1990] and more recently Kilburn et al. [1993], applying an interferometric (FDI) method developed by Kudeki and Stitt [1987] with a 10-m resolution to the MU radar, observed many stratospheric layers with a thickness of the order of $50-100 \mathrm{~m}$.

Warnock et al. [1978] pointed out that these turbulent layers are advected by the wind. Then, the horizontal extent of these structures can be deduced from radar observations of their lifetime. Using the Chatanika 1290-MHz radar, Balsley and Peterson [1981] estimated an horizontal extent larger than several kilometers. Their observed lifetime has been observed to vary from a few minutes up to a few hours, which is an indication of a large but variable horizontal extent.

In these layers the refractive index (or temperature) fluctuations with scales lower than about $10 \mathrm{~m}$ (the buoyancy scale) are supposed to be only weakly influenced by the stratification and are then supposed to be essentially isotropic [Hocking, 1985]. From dimensional arguments the $-11 / 3$ law of the 3D spectral power density (or 3D spectrum) of refractive index fluctuations is obtained in the inertial subrange [Tatarski, 1961]. Under some general conditions [Doviak and Zrnic', 1984], radar measurements give access to this 3D spectrum at the half radar wavelength. Measurements in 


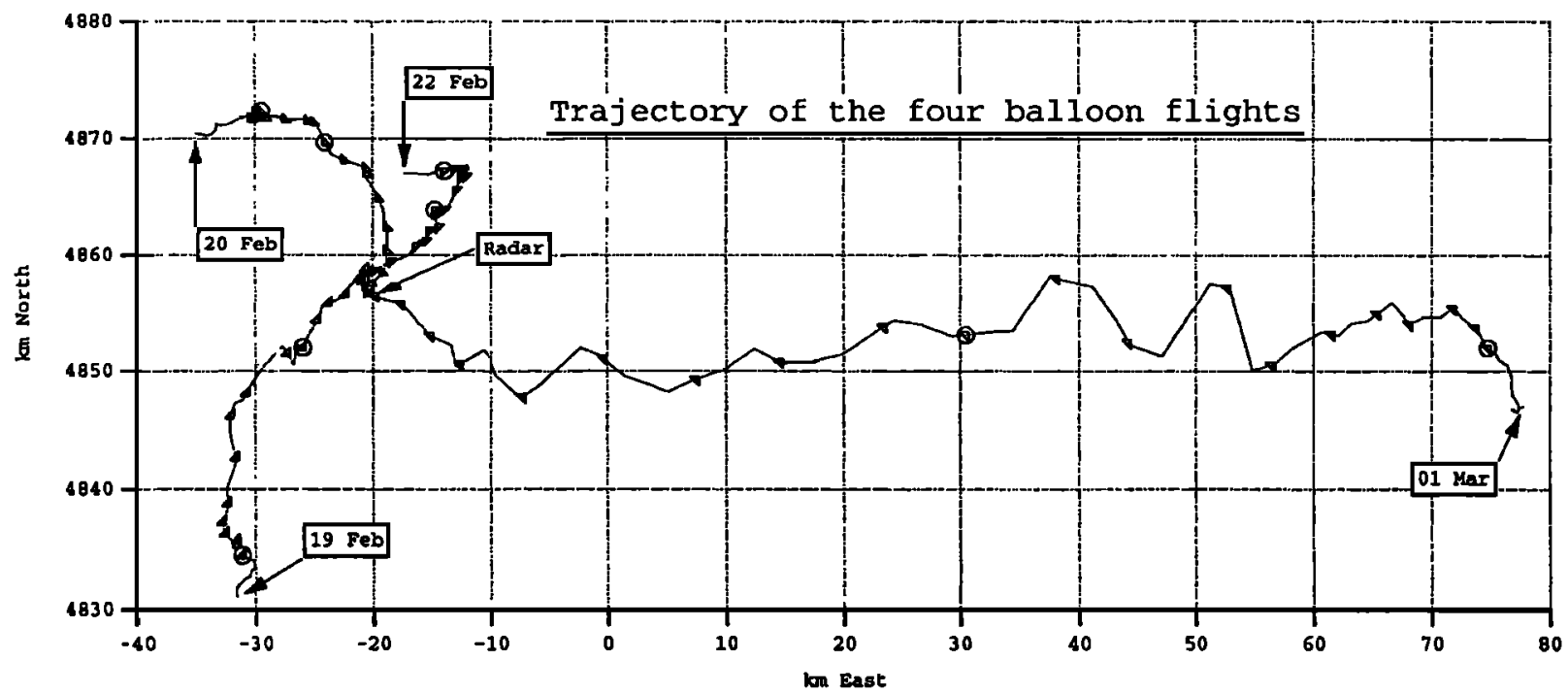

Figure 1. Ground projection of the trajectory of the four balloon flights during the ascent. Altitude is indicated by a triangle every kilometer and a circle every $10 \mathrm{~km}$. The location of the radar is indicated by the arrow. It can be noted that the first three balloons stay around the radar site. The maximum horizontal separation is typically lower than 15 $\mathrm{km}$. In contrast, on March 1, the balloon rapidly goes away from the radar because of the presence of a strong jet.

different directions with respect to the zenith, allow investigations of the possible 3D spectrum anisotropy at a given wavelength [e.g., Tsuda et al., 1986; Hocking et al., 1990; Yoe et al., 1994]. The 3D spectrum evaluation for a given direction and different wavelengths needs the use of perfectly (inter-) calibrated radars. This is technically difficult and expensive. For these reasons a complete description of the 3D spectrum has not yet been obtained using radar measurements. However, Rogers et al. [1992] made radar measurements in the first $3 \mathrm{~km}$ at 5 and $33 \mathrm{~cm}$ and showed that the scattering process hypothesis from inertial isotropic turbulence seems to be well adapted to explain the radar echoes at these scales. More recently, Cohn [1994] compared measurements obtained for UHF (440 MHz), L (1.3 GHz) and $\mathrm{X}(10 \mathrm{GHz})$ bands in the troposphere and showed that the $-11 / 3$ dependency of the 3D spectrum was generally observed. The CAT investigation at these frequencies is often difficult because of the strength of the hydrometeor echoes. Röttger [1986] established that the measurements in oblique $6.5^{\circ}$ and $12^{\circ}$ directions, at $46.8 \mathrm{MHz}$ and $430 \mathrm{MHz}$, respectively showed similar echo strength variations with altitude. This observation indicates that the same structures should be at the origin of the echoes. Other measurements in the mesosphere at 2.66 and 40.92 MHz [Royrvik, 1985] put forward that scattering from turbulence was at the origin of the echoes for these two wavelengths, whereas Hocking and Vincent [1982] proposed specular reflection as a source of the echoes below $80 \mathrm{~km}$ at 2 and $6 \mathrm{MHz}$.

The $-11 / 3$ law of the 3D spectrum implies the wellknown $-5 / 3$ law of the one-dimensional (1D) spectrum observed from in situ measurements. Indeed, such a spectral law may be observed in some scale range, at least when sufficiently wide atmospheric layers including turbulent patches are considered (see Figure 2). However, as a result of stable stratification, larger (vertical) scales are more and more influenced by buoyancy forces. Then, energy spectra scaling like $k_{z}^{-3}$ are observed, in the so called "turbulent-buoyancy subrange", and this effect is also evident in the potential energy (temperature) spectra [Dalaudier et al., 1994b]. Schematically, the -3 law of the $1 \mathrm{D}$ spectrum is associated with the stratification of the medium, the spectral level being correlated with the stability [Lumley, 1964; Weinstock, 1978, 1985; Holloway, 1986].

\section{Reconstruction of the Oblique Echo Profiles Using Independent Data Sets}

\subsection{Indirect Attempts}

A few reconstruction attempts relying upon the same theoretical background have been published. Some of them use an independently acquired statistical charac- 


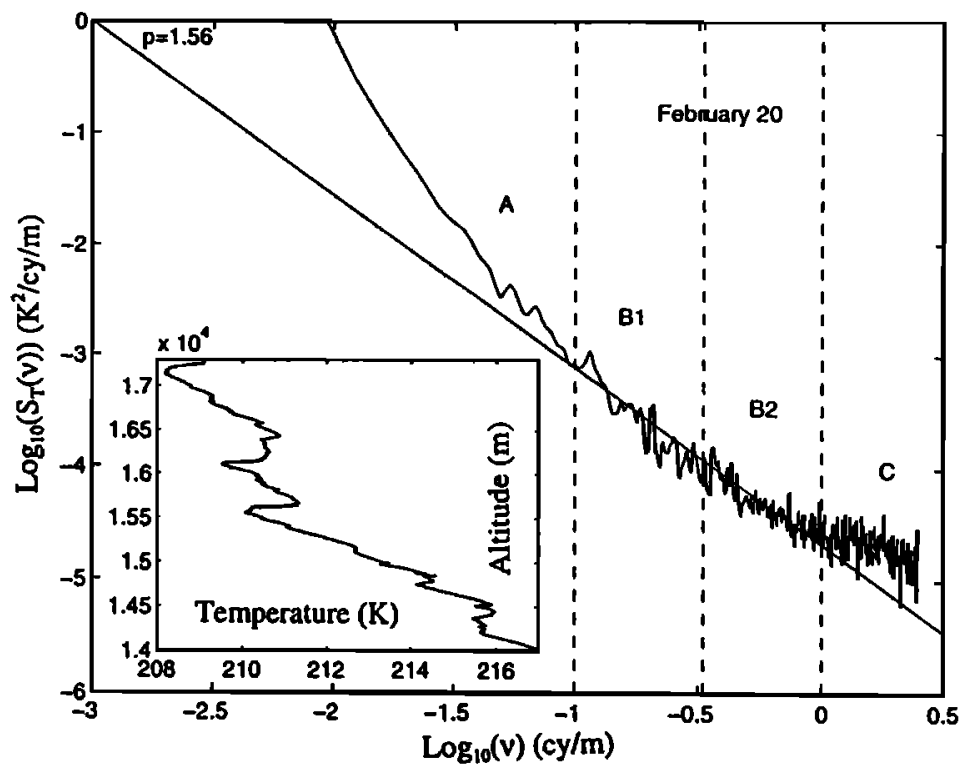

Figure 2. Estimation method of the spectral slope and level at the Bragg wavenumber. The method is exemplified using here a typical temperature spectrum measured in a wide stratospheric region, including turbulent and nonturbulent layers; the corresponding temperature profile is shown: notice that turbulent fluctuations are nearly invisible. A succession of distinct spectral domains with spectral slopes close to -3 (band A), $-5 / 3$ (band B), and 0 (noise limit, band C) may be observed. Assuming that each local spectrum ( 50 -m-wide data section) presents the form $a k^{p}(\mathrm{p}<0)$, the method consists in determining $a$ and $p$ by the calculation of the variance in two spectral bands (B1 and B2) of identical relative logarithmic width $\Delta k / k \approx 1$. The value $S_{T}\left(k_{b}\right)$ is then determined at $k_{b}$ by calculating $a k_{b}^{p}$.

terization of the field in order to infer the radar returns. They are termed here "indirect," as distinct from those using actual fluctuation measurements, termed "direct."

1. Van Zandt et al. [1978] elaborated upon a statistical model of $C_{n}^{2}$ based on the probability of existence of homogeneous turbulent layers when the Richardson number, $R i$, obtained by classical radiosonde data with a range resolution of $100 \mathrm{~m}$, is lower than $1 / 4$. This model was compared with $C_{n}^{2}$ radar profiles with a range resolution of $1 \mathrm{~km}$ measured at $40.5 \mathrm{MHz}$ and $30^{\circ}$ from the zenith. Despite a large time variability of the radar profiles (about $20 \mathrm{~dB}$ for 1 hour), the results agreed well in shape and in dynamic range. However, this agreement was obtained only with a relative level since the external scale of the turbulence, $L_{0}$, was used as an adjustable parameter.

2. Comparisons of $C_{n}^{2}$ measured by VHF radars and scidar (scintillation detection and ranging) were made on several occasions [e.g., Green et al., 1984]. The scidar is an optical (passive) instrument used to estimate not only the quality of seeing (for astronomical observations) but also the intensity and the altitude of the atmospheric turbulent layers. Radar and scidar are sensitive to different statistical characteristics of the refractive index fluctuating field: the value of the $3 \mathrm{D}$ spectrum at half radar wavelength and the value of an autocorrelation coefficient at a horizontal separation of a few tens of centimeters. The experiment described by Vernin et al. [1990] used the scidar of the Département d'Astrophysique de Nice and the 45-MHz "Provence" radar of the LSEET (Laboratoire de Sondages Electromagnétiques de l'Environnement Terrestre). Many similarities were observed and the discrepancies well explained (especially in the troposphere). However, the "radar $C_{n}^{2 " 1}$ was $6 \mathrm{~dB}$ lower on average than the "scidar $C_{n}^{2}$," and the difference was attributed to a calibration inaccuracy of the radar system.

It can then be concluded that these two attempts seem to support their common underlying hypotheses on dominant scattering by isotropic turbulence in the inertial subrange.

\subsection{A First Direct Attempt: The "Puzzling Result"}

Another approach was based on a direct comparison between in situ and radar measurements of temperature 
fluctuation spectra performed during the FRONT84 campaign [Dalaudier et al., 1989]. This method tried to use direct in situ estimates of $1 \mathrm{D}$ temperature spectra at the scale of one half radar wavelength based on simultaneous and nearly colocated balloon high-resolution measurements. This previous attempt lead to a "puzzling result," as indicated in the title of Dalaudier et al's paper. Neither the shape nor the dynamic range of the echo strength was correctly reproduced. Furthermore, the power level was strongly overestimated, typically by 15 dB. Following the much better results obtained in the present work, both the previous data sets and their processing were carefully and recently reexamined. Some inaccuracies and errors were found allowing us to explain clearly the causes of the previous discrepancies.

On the side of radar measurements, the power calibration was inaccurate. The new calibration is based on (1) various comparisons with scidar measurements [Vernin et al., 1990] and further measurements during the RASCIBA90 campaign and on (2) a reexamination of absolute calibration of radar system parameters. Compared to calibration used by Dalaudier et al. [1989], the atmospheric reflectivity deduced from radar measurements should have been increased by $6 \mathrm{~dB}$. The parameter recalibration, by itself, accounts for 3 to $4 \mathrm{~dB}$, while radar-scidar comparisons point to a 6-dB reevaluation (see section 4.1). Taking into account the absolute calibration uncertainties, these various figures are compatible within the error bars. Because the scidar $C_{n}^{2}$ profiles are supposed to be absolutely calibrated [e.g., Vernin et al., 1990], it was finally chosen to use the 6-dB correction. Furthermore, this value produces more consistent results for both oblique comparisons (this present work) and vertical comparisons [Dalaudier et al., 1994a; Luce et al., 1995].

On the side of in situ measurements, the "puzzling result" for the oblique reconstruction can be tracked down to two main causes: an important overcorrection for the thermistor time constant and probable contaminations of the temperature signal by wakes from various elements above or at the gondola level.

The overcorrection of the time constant accounts for a 6- to 9-dB (depending on the altitude) overestimate of the reconstructed $C_{n}^{2}$ levels. The contamination by a weak wake, by introducing a background of self-induced turbulence, explains the poor dynamic range of the oblique reconstruction. Each problem will be now examined in more detail.

Since the time constant of the thermistor $\tau$ was estimated to vary between 0.3 and $0.5 \mathrm{~s}$, depending on the altitude, a correction of the form $1+(\omega \tau)^{2}$ was applied to the spectral levels deduced from in situ measurements ( $\omega$ is the angular frequency). However, the time constant of the thermistor was strongly overestimated because (1) the diameter of the thermistors was (erroneously) used instead of its radius in the theoretical formulas for $\tau$ and (2) the effect of the lead wires between thermistor and mounting was not taken into account (because Dalaudier et al. [1989] were not aware of the potential importance of the lead wires for the thermal transfer). The error on the radius accounts by itself for 4 to $6 \mathrm{~dB}$ in the overestimation. The importance of the energy exchange through the lead wires has been recently emphasized and modeled by Fuehrer et al. (1994). Using their approach, it may be shown that the neglect of the lead wires account for 2 to $3 \mathrm{~dB}$ more. Both errors increase with altitude. It is worth noting that taking into account the thermal transfer through the lead wires improves significantly the transfer function of the thermistor. However, the resulting transfer function cannot any more be described as resulting from a time constant. Since the spectral level was estimated in a relatively narrow band $(\Delta v / v=0.4)$, corresponding to the half radar wavelength (around 1 $\mathrm{Hz}$ ), the calculation of the full transfer function, following the work of Fuehrer et al. [1994], corrected by Dalaudier [1996], and using the correct geometry for the thermistor, shows that no spectral correction was necessary in this frequency band. In other words, no spectral correction should have been applied to the data.

In relation to the low dynamic range of the reconstructed profiles, the detailed examination of the data obtained during the four flights of the RASCIBA90 campaign (present paper) clearly shows the presence of short periods during which the temperature data are (weakly) contaminated by nonatmospheric fluctuations. Such contamination arises as a consequence of the wake from the instrument itself (when its upwind orientation is not properly maintained, especially in the case of weak relative horizontal wind between the gondola and the balloon) or from cables or technical gondola located above the instrument. For the RASCIBA90 campaign data, the contaminations were identified and removed as far as possible (by "manual" scrutiny of the data). Unfortunately, such "decontamination" could not be performed on the FRONT84 data. Furthermore, because of its different geometry and mass distribution, the FRONT84 gondola was not always properly pointed upwind during its ascent, as already mentioned by Dalaudier et al. [1989]. Consequently, a "background" of contamination was probably present in the data. This 
background can account for the bad dynamical range of the reconstructed signal and also possibly for the poor shape agreement of the profiles.

Taking into account both radar and balloon corrections and errors, it can be concluded that the puzzling result obtained by Dalaudier et al. [1989] for oblique measurements is now understood. The new and more successful attempt of direct comparisons using new data obtained during the RASCIBA90 campaign is now presented.

\section{Experimental Setup and Accessible Parameters Using the Isotropic Hypothesis}

The RASCIBA90 campaign was performed in order to investigate the fine-scale structures of the troposphere and lower stratosphere, as viewed simultaneously by in situ balloon-borne sensors and remote sensing instruments. This experiment took place at the Centre National d'Etudes Spatiales (CNES) balloon launching site of Aire sur $1^{\prime}$ Adour $\left(43^{\circ} 42^{\prime} \mathrm{N}, 0^{\circ} 15^{\prime} \mathrm{W}\right)$ at the end of February and the beginning of March 1990 . The balloon instrumentation was developed by the Service d'Aéronomie du CNRS (Centre National de la Recherche Scientifique) while the humidity data set was provided by the Département d'Astrophysique de l'Université de Nice. Four balloon flights took place, on February 19, 20 , and 22 and on March 1. The ground projection of the trajectory of the four balloon flights is given in Figure 1. The horizontal separation between the radar and the balloon, which is less than $20 \mathrm{~km}$ for the three first flights, rapidly exceeds $30 \mathrm{~km}$ for the fourth launching, made during strong jet stream conditions (March 1).

Simultaneously, the "Provence" 45-MHz ST radar, operated by the LSEET and located near the launching site, allowed an exploration of the same atmospheric altitude range. A short account of the instrumental set up and of the measured parameters is given below.

\subsection{Radar Measurements}

The VHF 45-MHz "Provence" radar uses two 50-by-50 $\mathrm{m}$ antennas, each one composed of 16 strings of coaxial-collinear antennas. The antenna beam could be scanned sequentially between the vertical and two oblique directions $\left(15^{\circ}\right.$ south-east and south-west of the zenith). Its pulse peak power $P_{t}$ was $36 \mathrm{~kW}$, and the range resolution $\Delta r$ used during the campaign was $600 \mathrm{~m}$.

The refractive index 3D spectrum at the half radar wavelength scale is accessible from the radar measurements by using the following radar equation [Van Zandt et al., 1978; Doviak and Zrmic', 1984]:

$$
\frac{S}{N}=\frac{\alpha P_{r} A_{e}}{c K_{B}\left(T_{N}+T_{s} / \alpha\right)}\left(\frac{\Delta r}{r}\right)^{2} 4 \pi\left|\mathrm{k}_{r}\right|^{4} N_{C I} \Phi_{n}\left(2 \mathrm{k}_{r}\right)
$$

where $\alpha \approx 0.2$ is the efficiency factor for antenna and transmission lines, $A_{e}$ is the effective antenna area of the order of $1100 \mathrm{~m}^{2}, T_{N}$ is the cosmic noise temperature evaluated to $6000 \mathrm{~K}$ by Lamy et al. [1991], $T_{s} \approx 700 \mathrm{~K}$ is the temperature system noise including receiver and duplexor noise, $r$ is the radial range, $N_{C l}$ is the number of coherent integrations, $K_{B}$ is the Boltzman constant, $c$ is the velocity of light, $\mathbf{k}_{r}$ is the radar wave vector, and $\Phi_{n}$ is the mean 3D spectral power density of refractive index fluctuations in the sounded volume. The given radar parameters take into account the 3-to 4-dB recalibration (see section 3.2) with respect to the calibration given by Dalaudier et al. [1989]. These values do not consider the 2- to 3-dB reevaluation leading to the 6-dB correction used for the comparisons.

In fact, (1) is a simplified expression of the radar equation. The power received by a radar is proportional to the 3D spectrum of refractive index convolved with a spectral sampling function (SSF) taking care of the effect of the finite scattering volume [Doviak and Zrnic', 1984, see also Hocking and Röttger, 1983]. Because of the poor radial radar resolution, the radial effect of the convolution by the spectral sampling function can be neglected. Taking account of the transverse convolution is necessary if the backscattering process is produced by strongly anisotropic fluctuations. In other words, anisotropic models must include the transverse weighting function by introducing a convolution with the antenna beam pattern in the radar equation. Dalaudier et al. [1989] developed an anisotropic 3D model using a simplified expression for the radar lobe and showed that a moderately anisotropic turbulent component could contribute at $14.5^{\circ}$ from the zenith. They also showed that the narrower the radar lobe, the smaller the contribution.

Here, the isotropic scattering hypothesis will be used again. Physical reasons may be found in: (1) the lack of VHF radar aspect sensitivity for zenithal angles larger than $\approx 10^{\circ}$, as already mentioned; (2) the known fact that taking into account admitted values of both turbulent kinetic energy dissipation rates and stratification parameter within turbulent layers, the VHF radar Bragg wavelength is smaller than the Lumley-Shur (buoyancy) scale; this scale is expected to fairly represent a transition above which the (anisotropic) influence of stratification 


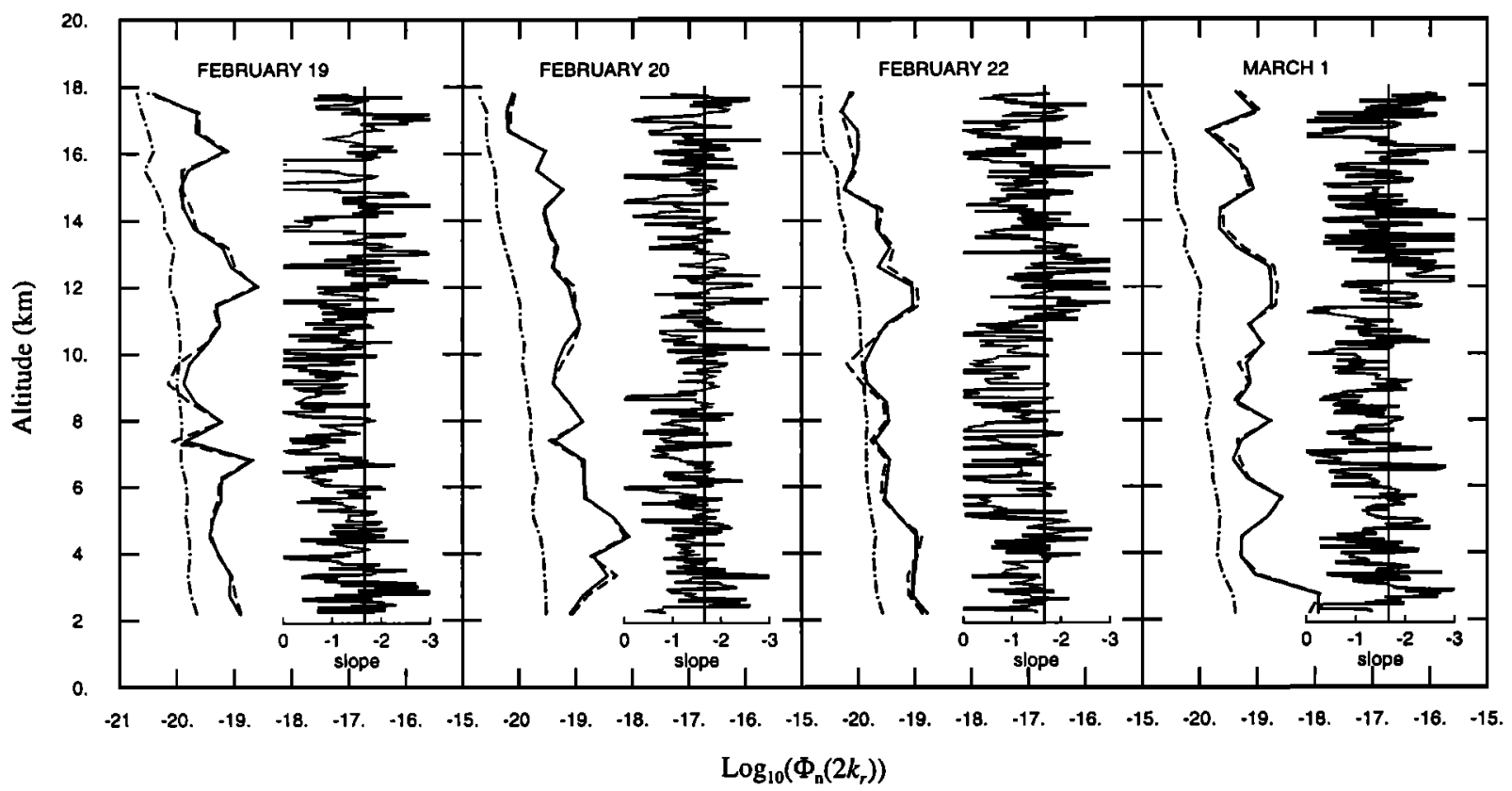

Figure 3. Comparison between the reconstructed profiles using the $-5 / 3$ and the observed slopes. Vertical profiles of $\Phi_{n}\left(2 k_{r}\right)$ in logarithmic scales deduced from temperature and humidity measurements at the radar resolution $(600 \mathrm{~m})$. The four panels correspond to the four flights measurements. The reconstructed profile with the $-5 / 3$ slope is given by the solid line, and the observed slope is shown by dashed line. The noise level is given by the thin dashed-dotted line. The two profiles are very similar. The computed slopes on sections of about $400 \mathrm{~m}$ thickness every $50 \mathrm{~m}$ are presented at the right of each profile. The $-5 / 3$ slope is given as reference by the vertical solid line.

should play a major role [Hocking, 1985]; (3) the consistency, within turbulent layers and in the vicinity of VHF radar Bragg wavenumbers, of our horizontal and vertical relative wind $U^{\prime}$ and $W^{\prime}$ spectra obtained from anemometric measurements made during the experiment (see section 4.2) with those predicted by the Kolmogorov theory. However, we emphasize that the slopes of local temperature spectra often depart from the expected $-5 / 3$ law, as shown in Figure 3, an observation which does not imply anisotropy of the temperature fluctuation field.

On the other hand, 1D/3D spectral conversions, an essential step for the balloon/radar comparisons, require some supplementary hypotheses about the 3D spectrum itself, which is nearly unknown. The choice of any anisotropic model (for instance, outside turbulent layers) would be highly arbitrary and would require supplementary parameters. This is why we used, everywhere, the isotropic model.

The oblique $\left(15^{\circ}\right)$ radar profiles will be presented here in terms of $\Phi_{n}\left(2 k_{r}\right)$. The detectability profile discriminating the signal from the noise will be plotted for each radar profile. This notion has already been used by, for example, Gage and Balsley [1978].

\subsection{Balloon Measurements}

Atmospheric temperature measurements were obtained during four balloon flights with a good vertical resolution (a function of the balloon ascent velocity but slightly less than $20 \mathrm{~cm}$ ). These measurements were performed with high-resolution (5-7 $\mathrm{mK}$ ) fast response platinum cold wire thermometers (time constant less than $10 \mathrm{~ms}$ ) which much improved upon the previously used microbead thermistor. Using the altitude time series deduced from high resolution pressure sensors (sampling rate $=1 \mathrm{~Hz}$, r.m.s. noise $=0.16 \mathrm{~Pa}$ ), the original temperature time series have been resampled (spline cubic interpolation) every $20 \mathrm{~cm}$, corresponding to a slight undersampling. Three thermometers were used, 1 $\mathrm{m}$ apart horizontally and vertically. Furthermore, anemometric data sets were obtained with a two-axis ionic anemometer [Barat, 1982]. From these measurements, mean wind profiles as well as turbulent velocity components can be computed. Humidity measurements were 
also available for three of the four flights (February 20 and 22 and March 1).

1D spectrum of temperature fluctuations. The 1D power spectral density of temperature, or 1D spectrum, $S_{T}(k)$, is accessible from balloon measurements with the Taylor (frozen field) hypothesis along the trajectory of the balloon. This $1 \mathrm{D}$ spectrum is related to the 3D spectrum $\Phi_{T}(\mathrm{k})$ by integration along two symmetric planes with respect to the origin, at a distance of $2 k$ and perpendicular to the velocity direction. Dalaudier et al. [1989] showed that the observed 1D vertical spectrum is a poor indicator of the anisotropy of the fluctuations since the ratio between the 1D spectrum produced by strongly anisotropic fluctuations and the one produced by isotropic fluctuations is equal to the scalar spectrum slope.

The vertical 1D spectrum can be defined as

$$
S_{T}^{z}\left(k_{z}\right)=2 F_{T}^{z}\left(k_{z}\right)=2 \iint_{\mathscr{x}^{2}} \Phi_{T}\left(k_{x}, k_{y}, k_{z}\right) d k_{x} d k_{y}
$$

where $F_{T}^{z}\left(k_{z}\right)$ is the two-sided 1D spectrum, $S_{T}^{z}\left(k_{z}\right)$ being defined for $k_{z}>0$ [Ottersten, 1969b]. However, the 1D estimated spectra given by sensors hung below a rising balloon are not purely vertical because of the relative horizontal velocity component. This velocity (most of time smaller than the vertical ascent velocity of the balloon) must be taken into account in the calculations. If $S_{T}^{\alpha}(k)$ (where $\alpha$ is the angle between the vertical axis and the relative velocity vector) is the estimated spectrum from the temperature data vertically resampled at a spatial constant step $(20 \mathrm{~cm})$, it can be easily shown that the vertical $1 \mathrm{D}$ spectrum is connected to the $1 \mathrm{D}$ spectrum along the gondola trajectory by

$$
S_{T}^{z}(k)=S_{T}^{\alpha}(k / \cos \alpha) / \cos \alpha
$$

Relation between 1D and 3D temperature spectra. Within the framework of the isotropic fluctuation hypothesis, a simple relation between the 3D spectrum and the 1D spectrum can be obtained [Ottersten, 1969b]:

$$
\Phi_{T}(k)=-\frac{p}{4 \pi} k^{-2} S_{T}^{z}(k)
$$

since $\Phi_{T}(\mathrm{k})=\Phi_{T}(k, \theta, \phi) \equiv \Phi_{T}(k)$ and $p(p<0$, required condition for isotropy) is the $1 \mathrm{D}$ spectrum slope $S_{T}^{z}(k)-k^{p}$. The particular case of the inertial range implies $p=-5 / 3$.

Conversion of the parameters expressed in terms of refractive index $n$. At radar frequencies and without ionization and humidity (as can be assumed in the stratosphere), the refractive index $n$ is only a function of the temperature $T(\mathrm{~K})$ and atmospheric pressure, $p_{r}(\mathrm{~Pa})$ :

$$
n-1=\frac{0.776 \times 10^{-6} p_{r}}{T}
$$

The 3D temperature and refractive index spectra are associated by the relation [Tatarski, 1961]

$$
\Phi_{n}(k)=\left(\frac{0.776 \times 10^{-6} p_{r}}{T^{2}}\right)^{2} \Phi_{T}(k)
$$

\section{Data Processing for Direct Comparisons}

The reconstruction method based on the isotropic scattering hypothesis uses the theory developed in the previous section. The method estimates the $3 \mathrm{D}$ isotropic spectrum of refractive index fluctuations at the $\lambda_{r} / 2$ radar scale, $\Phi_{n}\left(2 k_{r}\right)$, from in situ measurements. A first variant of this method needs the knowledge of the local slope of the 1D spectrum of temperature and its level at $\lambda_{r} / 2$. The second one (used by Dalaudier et al. [1989]) is based on the use of the $-5 / 3$ inertial slope compatible with the one supposed for the small scales.

\subsection{Humidity Contribution in the Troposphere}

The humidity effects in the reconstruction must be taken into account in the troposphere. These effects can be preponderant up to $8 \mathrm{~km}$ [e.g., Van Zandt et al., 1978; Tsuda et al., 1988; May et al., 1991]. An estimate of the potential refractive index gradient $M$ was made with and without humidity by using the relations given by Ottersten [1969a]:

$$
\begin{gathered}
M_{\text {wet }}=-0.776 \times 10^{-6} p_{r} T^{-2}\left(1+15600 q T^{-1}\right) \times \\
\left(\frac{d T}{d z}+\Gamma_{a}-\frac{7800}{1+15600 q T^{-1}} \frac{d q}{d z}\right)
\end{gathered}
$$

and

$$
M_{d r y}=-0.776 \times 10^{-6} p_{r} T^{-2}\left(\frac{d T}{d z}+\Gamma_{a}\right)
$$

With $q$, specific humidity $(\mathrm{kg} / \mathrm{kg}$ ) which represents the content of water vapor in the air and $\Gamma_{a}=-9.8 \times 10^{-3}$ degrees $/ \mathrm{m}$ is the adiabatic lapse rate. For a dry atmosphere, $M_{\mathrm{dry}}$ is proportional to $N^{2}$. 
Moreover, Tatarski [1961, p. 57] showed that the intensity of the refractive index fluctuations is proportional to $M$. Then, it can be shown by using the isotropic condition at $\lambda_{r} / 2$ that

$$
\Phi_{n}\left(2 k_{r}\right)_{\text {net }}=\frac{M_{u e t}^{2}}{M_{d r y}^{2}} \cdot \Phi_{n}\left(2 k_{r}\right)_{d r y}=R \cdot \Phi_{n}^{2}\left(2 k_{r}\right)_{d r y}
$$

The ratio $R$ was calculated for three profiles corresponding to balloon flights for which humidity measurement was available. The contribution of the humidity for the reconstructions will be shown to be important and discussed when the comparisons between reconstructed and observed profiles are presented.

\subsection{Estimate of the 3D Spectrum of Refractive Index at the $\lambda_{r} / 2$ Scale}

From the 1D vertical temperature spectra obtained from balloon measurements, the $3 \mathrm{D}$ refractive index spectrum profile for the $\lambda_{r} / 2$ scale, assumed to be measured by the radar in oblique direction $\left(15^{\circ}\right)$, is calculated. The method estimates local parameters (slope and level for the radar half wavelength) of the 1D vertical spectrum. However, because of the horizontal separation, sometimes large, between the balloon and the radar, it is unrealistic to suppose that for a given altitude range, the estimated parameters correspond effectively to those observed by radar. It is assumed instead that the fluctuations measured by the balloon sensors are representative (in a statistical sense) of the fluctuations observed by the radar. In other words, it is assumed that given the range radar resolution, the mean atmospheric conditions leading to the formation of turbulent layers extend horizontally much further than the radar-balloon horizontal distance. It is also assumed that the altitude variations of the layer do not exceed the range resolution and that the observed temperature fluctuation intensity is representative of the intensity measured by radar. If this is verified, the observed spectral slopes could be more significant in order to reproduce the radar measurements than the $-5 / 3$ slope. The use of the $-5 / 3$ slope instead of the locally observed one (method reduced to one parameter) corresponds to the empirical observation that "on an average" the local slope leads to this value.

First, the 1D temperature spectra are estimated for sections of about $50 \mathrm{~m}$. The spectral slope is then obtained around $\lambda_{r} / 2$ by calculating the variance in two frequency bands of identical relative (logarithmic) width
$\Delta k / k \approx 1$. In practice, the scale range used is (1...10) meters and was chosen with the idea that it corresponds roughly to the assumed inertial subrange within turbulent layers. Indeed, the small-scale cutoff is introduced in order to avoid a possible underestimate of the slope produced by the spectral density of the white noise at high frequencies. The upper limit is introduced in order to restrict the scales domain used for the estimate to the vicinity of the radar half wavelength.

The spectral level $S_{T}(k)$ for $k_{b}=2 k_{r}$ is calculated as indicated in Figure 2 (by taking the value on the straightline, in log-log coordinates, leading to the same variances as those measured in the two bands between 1 and $10 \mathrm{~m}$ ) and by taking into account the relative horizontal wind correction (relation 3 ). The spectral level corresponding to the white noise $(\approx 5$ to $7 \mathrm{mK})$ is then subtracted. This noise level, variable with altitude, was estimated by calculation of the variance in a highfrequency band of the spectrum corresponding to the scales smaller than $1 \mathrm{~m}$ (see Figure 2). For these scales it was first supposed that the spectral fluctuations are due only to the white noise. However, in regions of high turbulent patches, high-frequency atmospherical signals can overestimate the mean variance of the noise. In this case the estimated variance was replaced by a more realistic value (as far as possible) deduced from spectra of nearby quiet regions.

The 3D spectral level is then deduced from the relation (4) by using either the observed spectral slope or the $-5 / 3$ one. Vertical profiles are thus obtained with a $50-\mathrm{m}$ resolution. In order to compare with the radar profiles, they are smoothed using the height weighting function of the radar. Finally, the humidity contribution is taken into account when available by using (9).

Comparisons between variants of the method (observed and $-5 / 3$ spectral slope) are shown in Figure 3. The noise level of the reconstruction and the spectral slope profiles of the 1D temperature spectra are also given. It can be seen that the profiles are very similar. However, the "-5/3" choice shows a tendency to produce a higher level in regions of weak activity (generally between 8 and $10 \mathrm{~km}$ ) and is less important in very stable regions (around $12 \mathrm{~km}$ for example). This observation can be understood since the observed slope tends respectively toward zero (because of the preponderant instrumental noise) and is steeper than $-5 / 3$ (because of the contribution of stable layers producing a -3 slope). However, it is interesting to note that the 1D spectral slope is not critical and cannot be a cause of a real discrepancy for the comparisons with the radar profiles. 
Although the method of slope estimation can be at the origin of some dispersion since the inertial slope can be observed only if the inertial subrange is dominant at least until $10 \mathrm{~m}$, the slope profiles show that the $-5 / 3$ slope is rarely observed. The very small values (between 0 and -1) are principally due to the noise level and the steeper ones are generally associated with regions of high stability. It can be noted that the slope profile is more uniform on February 20 and the inertial slope is more frequently observed especially in the stratosphere.

\subsection{Details of the Experimental and Processing Improvements}

1. With respect to the experiment described by Dalaudier et al. [1989], more extensive in situ and radar data sets are now available. Four comparisons with data sets obtained during both quiet and disturbed meteorological conditions are now possible. The model used can then be tested for different conditions.
2. On the side of radar measurements, the power calibration has been improved, and a much larger number of vertical profiles are available ( 8 to 10 for each comparison). In order to identify regions where the radar signal can be meaningfully compared with in situ measurements, the detectability threshold [Gage and Balsley, 1978] calculated as by Ferrat and Crochet [1994] is plotted in Figure 4 along with the radar signal using the same units.

3. Concerning the in situ measurements, several improvements can be noted: (1) The measurements were performed during the night in order to avoid any radiative contamination of the sensors [Dalaudier et al., 1994a]. (2) The gondolas are smaller and lighter than the gondolas used by Dalaudier et al. [1989]. Their orientation with respect to the wind was then better and faster, allowing fewer disturbances by the wake from cables and the instrumentation itself. (3) The time response of the cold wire thermometers used during the RASCIBA90 campaign is sufficiently short in order to avoid

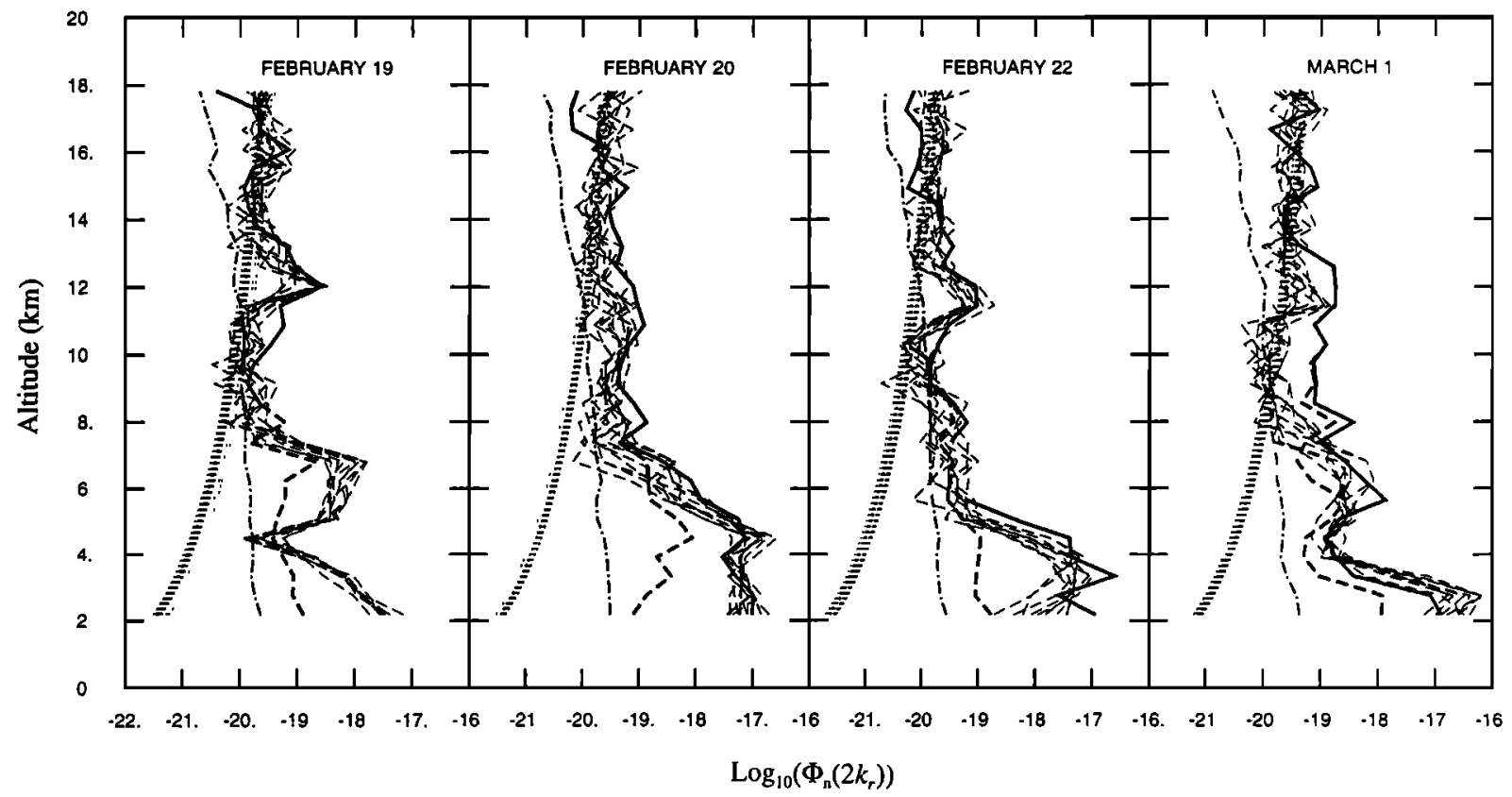

Figure 4. Comparison between radar observations and reconstructions from temperature measurements. Shown are vertical profiles, in logarithmic scales, of measured (thin dashed lines) and estimated (thick solid lines and thick dashed lines for the dry profiles) 3D spectrum of refractive index fluctuations (using a constant $-5 / 3$ slope). All the radar profiles obtained during the ascent of the balloons are given. The standard radar detectability threshold is indicated for each profile by the dotted lines (see text). The noise level of the reconstructions is given as a dashed-dotted thin line. Humidity measurements were not available on February 19. Data loss prevented humidity corrections on February 22 around $6.5 \mathrm{~km}$. A time variability of the radar measurements which is smaller than one decade can be observed. The three reconstructed profiles agree well in shape, and dynamic range and quite well in level with the observed ones. For the fourth flight the shape and dynamics are well reproduced but the level is strongly overestimated between 8 and $13 \mathrm{~km}$. 
any correction of the estimated 1D spectrum level (although the correction was unnecessary at $3 \mathrm{~m}$ scale for the data used by Dalaudier et al. [1989]; see section 3.2). (4) High-resolution temperature measurements are available in the troposphere and in the lower stratosphere (from the ground up to about $25 \mathrm{~km}$ ) whereas the temperature sensors used by Dalaudier et al. [1989] were saturated for altitudes lower than about $8 \mathrm{~km}$. (5) Humidity measurements obtained with Väissälä RS80 sondes and a 9-m sampling are available for three flights. These measurements allow us to take into account the contribution of the humidity in the troposphere by using the method described in section 5.1.

4. Concerning the data processing, some improvements are introduced: (1) The spectral slope of the 1D temperature spectrum is estimated based on the variances between 1 and $10 \mathrm{~m}$; two types of reconstructions are then performed in order to estimate the contribution of the observed slopes instead of just the $-5 / 3$ one. (2) The few regions contaminated by the wake of the instruments and balloons are carefully determined and suppressed without any ambiguity for the most important of them. (3) The noise level of temperature measurements is subtracted for each estimated temperature 1D spectrum. The noise level (smoothed at the radar resolution) is indicated on each of the four profiles. (4) The oblique path of the gondola with respect to the flow was taken into account in the temperature spectral estimate (relation (3)).

\section{Comparisons Between Radar Observa- tions and Reconstructions}

\subsection{Results Obtained on February 19, 20, and 22}

These 3 days correspond to quiet meteorological conditions with wind speeds typically lower than $10 \mathrm{~ms}^{-1}$ and a maximum horizontal separation between radar and balloon which was typically smaller than $25 \mathrm{~km}$ (Figure 1).

The general comparison between profiles reconstructed with a $-5 / 3$ slope and observed radar profiles shows a good general agreement (Figure 4).

1. The shapes are very similar. Particularly, the enhancements around $12 \mathrm{~km}$ are clearly reproduced.

2. The dynamic range of measured and reconstructed profiles is nearly the same. It can be noted that the suppression of the noise level (see section 5.2) contributes slightly to the improvement of the dynamic range especially in regions of low activities.

3. The use of the estimated slope instead of the expected one $(-5 / 3)$ has usually a very weak effect (see
Figure 3). However, in some regions of weak activity (February 19 around $9 \mathrm{~km}$, and February 22 around 10 $\mathrm{km}$ ), its use slightly improves the restitution of the weak observed echoes.

4. The reproduced level is quite good with a slight uncertainty due to the radar calibration.

5. Humidity data are available on February 20 and 22. The contribution of this parameter appears to dominate in the (lower) troposphere. The agreements confirm the importance of the humidity in this region of the atmosphere.

6. It is interesting to note that the level of the reconstruction is lower than the radar level when the backscattered power is too low to be detected (see the detectability threshold).

Some local disagreements (concerning one or two successive radar gates) can be observed on these quiet days: for example, overestimates are clear around $11 \mathrm{~km}$ on February 19.

On February 19, because the humidity data are not available, only dry refractive index reconstructions are plotted. It is worth noting that the level, shape, and dynamic range are quite well reproduced. The shape enhancement observed around $12 \mathrm{~km}$ is perfectly reconstructed by the model. In the stratosphere the signal level is generally below the detectable threshold, but there are significant and sporadic enhancements (fluctuating in time and height). The reconstruction indicates the same kind of enhancement in the same region around $16 \mathrm{~km}$ which points out the physical reality of (very probably) sporadic turbulent layers observed by the radar.

On February 20 a good agreement is observed between the radar profiles and the reconstruction. The low dynamic range above the lower troposphere presented by the radar measurements is reproduced. The power level is quite uniform in height but sufficiently high in order to be above the detectability threshold up to $14 \mathrm{~km}$, in contrast to the three other observations which do not show atmospheric signal around $10 \mathrm{~km}$.

On February 22 the agreement is noteworthy and noticeable discrepancies are not observed.

These results show a dramatic improvement respectively to Dalaudier et al.'s [1989]. They unambiguously support the isotropic turbulence model to reconstruct realistic oblique radar echoes profiles.

\subsection{Results Obtained on March 1}

This fourth night of experiment corresponds to measurements obtained during strong eastward wind conditions. A broad jet stream, with a wind velocity 
exceeding $45 \mathrm{~m} \mathrm{~s}^{-1}$ from 6 to $13 \mathrm{~km}$ height, carried away the balloon far from the radar site: the radar-balloon distance was already $50 \mathrm{~km}$ at a balloon altitude of 10 km (see Figure 1).

While consistent results are obtained below $8 \mathrm{~km}$, striking disagreements are observed between 8 and 13 $\mathrm{km}$ (the overestimate is typically $10 \mathrm{~dB}$ ). They could be attributed, at the first sight, to a horizontal inhomogeneity of the refractive index fluctuations during disturbed conditions possibly related to meteorological or orographic variations. However, this explanation is not convincing because the discrepancies always show an overestimate of the reconstruction. We can also suspect some contaminations of the measurements by turbulent wakes, contaminations that would be indistinguishable from real atmospheric turbulence, using the methods that proved to be efficient elsewhere. This interpretation is supported by the balloon anemometric data which show very weak horizontal relative winds (the wind shear on the balloon-gondola distance of $175 \mathrm{~m}$ ) within the jet and, consequently, many instabilities in the orientation of the gondola. From these data a mean wake-gondola distance may be computed. This distance appears to be of the order of one balloon diameter (about $20 \mathrm{~m}$ ) within most of the considered region. Thus contamination of the data by balloon and gondola wakes are highly probable. It may be noticed that the simultaneous observations of weak local velocity shears in the central part of a strong jet and of weak radar returns from the same area are fully consistent.

The agreement above $13 \mathrm{~km}$ is not significant since the radar measurements were below the sensitivity threshold.

\section{Conclusion and Perspectives}

The present work addresses the problem of the mechanism responsible for the VHF radar echoes in oblique directions. The method used is a direct comparison of the inferred $3 \mathrm{D}$ spectrum of refractivity fluctuations as determined from radar echo power and from simultaneous, nearly colocated, in situ measurements obtained from tropostratospheric balloon flights. The originality of this method is that both in situ and remote sensing measurements are performed at the same scale, corresponding to the half radar wavelength. The model used for this comparison is based on the standard hypothesis that the refractivity fluctuations responsible for the oblique radar echoes are produced by isotropic turbulence.
A previous attempt by Dalaudier et al. [1989] using the same method and hypothesis led to a "puzzling result" as indicated in the title of their paper. However, acareful reexamination of this previous work, in the light of the present comparison, allowed us to understand the stated discrepancies as a result of a conjunction of errors, inaccuracies, and technical problems (see section 3.2). On the other hand, the other "puzzling result" presented in that paper is now understood as a consequence of an inadequate modelling of the mechanism responsible for the vertical echoes [Dalaudier et al., 1994; Luce et al., 1995]. Consequently, we can state the previous puzzle is now completely solved.

Many experimental and data-processing improvements were performed with respect to that previous attempt (see section 5.3). The direct comparison shows a satisfying agreement between radar and in situ measurements for most of the available data. This agreement is obtained both in the troposphere and in the (lower) stratosphere without any adjustment between the two data sets. The shape and the dynamic range of the profiles are conveniently reconstructed, taking into account the experimental limitations coming from both radar and in situ noise levels. It is worth noting that the present agreement was obtained with a radar resolution of 600 $\mathrm{m}$. Such "moderate" resolution allows some smoothing of the in situ profile of the highly inhomogeneous turbulent fluctuations. This smoothing is interpreted as a statistical averaging of the atmospheric turbulence parameters. The averaged parameters are assumed to be nearly constant over horizontal distances of a few tens of kilometers.

The disagreement observed for part of the data (see section 6.2) is interpreted as the result of a contamination of in situ measurements by nonatmospheric turbulence. This contamination, which could not be distinguished from "true" turbulence, is attributed to the existence of very weak wind shears within the core of a strong jet. These weak shears are associated with very low levels of atmospheric turbulence as indicated by the simultaneous lack of oblique radar echoes.

The overall agreement of the present comparison allows us to conclude, as expected, that the scattering of radio waves by turbulent refractivity fluctuations is the dominant mechanism for the oblique echoes. The radar echo profile, with $600 \mathrm{~m}$ resolution, is well reconstructed from in situ estimations of the 3D spectrum of refractive index inhomogeneities. These estimations require the hypothesis of isotropy at the Bragg scale (here about 3 $\mathrm{m})$, and the comparison shows that the further hypothesis 
of inertiality (slope -5/3) can also be used. These successful reconstructions demonstrate the efficiency of the current physical model of radio wave scattering, even if they do not prove the validity of the underlying hypotheses.

We should note that in situ measurements with such a high resolution are difficult and that the 1D spectra analyzed here do not contain much information about the anisotropy of the observed structures. However, the use of three temperature sensors with vertical and horizontal separation of 1 meter will allow us to investigate further this question in subsequent analysis of the same data set.

Acknowledgments. We thank J. Vernin and M. Azouit from the University of Nice for their cooperation; C. Bourdier, J. Gagelli, and Y. Ruin from the LSEET for ST radar implementation on the site; and B. Lamy, J.L. Caccia, and E. Spano for experimental assistance. We thank J. C. Génie, Y. Nizou, $M$. Leclère, and $P$. Hulin for the technical achievement of the balloon gondola. We thank also all the balloon team from the CNES (Centre National d'Etudes Spatiales) and M. Faucon and Vincent from the CNES for allowing the ST radar implementation on the launching site and making all facilities available to the radar team. This program was supported by PAMOY (Programme Atmosphère Moyenne) and RADAR ST OPERATION from INSU-CNRS (Institut National des Sciences de l'Univers-Centre National de la Recherche Scientifique).

\section{References}

Balsley, B. B., and V. L. Peterson, Doppler-radar measurements of clear air atmospheric turbulence at $1290 \mathrm{MHz}$, J. Appl. Meteorol., 20, 266-274, 1981.

Barat, J., Some characteristics of clear-air turbulence in the middle stratosphere, J. Atmos. Sci., 39, 2553-2564, 1982.

Cohn, S. A., Investigations of the wavelength dependence of radar backscatter from atmospheric turbulence, J. Atmos. Oceanic. Technol., 11, 225-238, 1994.

Crane, R. K., A review of radar observations of turbulence in the lower stratosphere, Radio Sci., 15, 177-193, 1980.

Dalaudier, F., M. Crochet, and C. Sidi, Direct comparison between in situ and radar measurements of temperature fluctuation spectra: A puzzling result, Radio Sci., 24, 311-324, 1989.

Dalaudier, F., C. Sidi, M. Crochet, and J. Vernin, Direct evidence of "sheets" in the atmospheric temperature field, J. Atmos. Sci., 51, 237-248, 1994a.

Dalaudier, F., A. S. Gurvich, V. Kan, and C. Sidi, Middle stratosphere temperature spectra observed with stellar scintillation and in situ techniques, Adv. Space Res., 14, 961-964, 1994b.

Dalaudier, F., Correction to "Frequency response of a ther- mistor temperature probe in air," J. Atmos. Oceanic Technol., 13, 774, 1996.

Doviak, R. J., and D. S. Zmic', Reflection and scatter formula for anisotropically turbulent air, Radio Sci., 19, 325-336, 1984.

Ferrat, S., and M. Crochet, Methods of detection and estimation errors in ST radar studies, Ann. Geophys., 12, 489-496, 1994.

Fuehrer, P. L., C. A. Friehe, and D. K. Edwards, Frequency response of a thermistor temperature probe in air, J. Atmos. Oceanic. Technol., 11, 476-488, 1994.

Gage, K. S., and B. B. Balsley, Doppler radar probing of the clear atmosphere, Bull. Amer. Meteorol. Soc., 59, 1074-1093, 1978.

Green, J. L., J. Vernin, and W. L. Clark, A comparison of optical and radar measurements of $C_{n}^{2}$ height profiles, paper presented at 22nd Conference on Radar Meteorology, Am. Meteorol. Soc., Boston, Mass., 10-13 September, 1984.

Hocking, W. K., Measurement of turbulent energy dissipation rates in the middle atmosphere by radar techniques: A review, Radio Sci., 20, 1403-1422, 1985.

Hocking, W. K., and J. Röttger, Pulse length dependence of radar signal strengths for Fresnel backscatter, Radio Sci., 18, 1312-1324, 1983.

Hocking, W. K., and R. A. Vincent, A comparison between HF partial reflection profiles from the D-region and simultaneous Langmuir probe electron density measurements, J. Atmos. Terr. Phys., 44, 843-854, 1982.

Hocking, W. K., S. Fukao, T. Tsuda, M. Yamamoto, T. Sato, and S. Kato, Aspect sensitivity of stratospheric VHF radio wave scatterers particularly above $15 \mathrm{~km}$ altitude, Radio Sci., 25, 613-627, 1990.

Holloway, G., Considerations on the theory of temperature spectra in stably stratified turbulence, J. Phys. Oceanogr., 16, 2179-2183, 1986.

Ierkic, H. M., R. F. Woodman, and P. Perillat, Ultrahigh vertical resolution radar measurements in the lower stratosphere at Arecibo, Radio Sci., 25, 941-952, 1990.

Kilburn, C., S. Fukao, and M. Yamamoto, Frequency domain interferometry on the MU radar using two pairs of frequencies, paper presented at Sixth Workshop on Technical and Scientific Aspects of MST Radar, Sci. Comm. on Sol. Terr. Phys., Taiwan, August 17-20, 1993.

Kudeki, E., and G. R. Stitt, Frequency domain interferometry: A high resolution radar technique for studies of atmospheric turbulence, Geophys. Res. Lett., 14, 198-201, 1987.

Lamy, B., M. Crochet, and F. Dalaudier, Calibration of ST radars using cosmic noise evolution: Application to radar Provence at $45 \mathrm{MHz}$, paper presented at Fifth Workshop on Technical and Scientific Aspects of MST Radar, Sci. Comm. on Sol. Terr. Phys., U.K., August 6-9, 1991.

Luce, H., M. Crochet, F. Dalaudier, and C. Sidi, Interpretation of VHF ST radar vertical echoes from in situ temperature sheet observations, Radio Sci., 30, 1003-1025, 1995. 
Lumley, J. L., The spectrum of nearly inertial turbulence in a stably stratified fluid, J. Atmos. Sci., 21, 99-102, 1964.

May, P. T., M. Yamamoto, S. Fukao, T. Sato, S. Kato, and T. Tsuda, Wind and reflectivity fields around fronts observed with a VHF radar, Radio Sci., 26, 1245-1249, 1991.

Ottersten, H., Mean vertical gradient of potentiel refractive index in turbulent mixing and radar detection of C.A.T., Radio Sci., 4, 1247-1249, 1969a.

Ottersten, H., Radar backscattering from the turbulent clear atmosphere, Radio Sci., 4, 1251-1255, $1969 \mathrm{~b}$.

Palmer, S. J., R. F. Woodman, S. Fukao, M. F. Larsen, M. Yamamoto, T. Tsuda, and S. Kato, Frequency domain interferometry observations of tropo/stratospheric scattering layers using the MU radar: description and first results, Geophys. Res. Lett., 17, 2189-2192, 1990.

Rogers, R. R., C. A. Knight, J. D. Tuttle, W. L. Ecklund, D. A. Carter, and S. A. Ethier, Radar reflectivity of the clear air at wavelengths of 5.5 and $33 \mathrm{~cm}$, Radio Sci., 27, 645-659, 1992.

Röttger, J., P. Czechowsky, and G. Schmidt, First low-power VHF radar observations of tropospheric, stratospheric and mesospheric winds and turbulence at the Arecibo Observatory, J. Atmos. Terr. Phys., 43, 789-800, 1981.

Röttger, J., Comparison of reflectivity and wind profiles measured on $46.8 \mathrm{MHz}$ and $430 \mathrm{MHz}$ at the Arecibo observatory, Middle Atmos. Prog. Handb., 20, 307-308, 1986.

Royrvik, O., Radar comparison of $2.66 \mathrm{MHz}$ and $40.92 \mathrm{MHz}$ signals scattered from the mesosphere, Radio Sci., 20, 1423-1434, 1985.

Rüster, R., P. Czechowsky, and G. Schmidt, VHF radar measurement of dynamical processes in the stratosphere and mesosphere, Geophys. Res. Lett., 7, 999-1002, 1980.

Sato, T., and R. F. Woodman, Fine altitude resolution observation of stratospheric turbulent layers by the Arecibo $430 \mathrm{MHz}$ radar, J. Atmos. Sci., 39, 2553-2563, 1982.

Tatarski, V. I., Wave propagation in a turbulent medium, translated by R.A. Silverman, McGraw-Hill, New York, 1961.

Tsuda, T., T. Sato, K. Hirose, S. Fukao, and S. Kato, MU radar observations of the aspect sensitivity of backscat- tered VHF echo power in the troposphere and lower stratosphere, Radio Sci., 21, 971-980, 1986.

Tsuda, T., P. T. May, T. Sato, S. Kato, and S. Fukao, Simultaneous observations of reflection echoes and refractive index gradient in the troposphere and lower stratosphere, Radio Sci., 23, 655-665, 1988.

Van Zandt, T. E., J. L. Green, K. S. Gage, and W. L. Clark, Vertical profiles of refractivity turbulence structure constant: Comparison of observations by the Sunset radar with a new theoretical model, Radio Sci., 13, 819-829, 1978.

Vemin, J., M. Crochet, M. Azouit, and O. Ghebrebrhan, Scidar/radar simultaneous measuremnets of atmospheric turbulence, Radio Sci., 25, 953-959, 1990.

Warnock, J. M., T. E. VanZandt, J. L. Green, and R. H. Winkler, Comparison between wind profiles measured by Doppler radar and by rawinsonde ballons, Geophys. Res. Lett., 5, 109-112, 1978.

Weinstock, J., Vertical turbulent diffusion in a stably stratified fluid, J. Atmos. Sci., 35, 1022-1027, 1978.

Weinstock, J., On the theory of temperature spectra in a stably stratified fluid, J. Phys. Oceanogr., 15, 475-477, 1985.

Woodman, R.F., and A. Guillén, Radar observations of wind and turbulence in the stratosphere and mesosphere, $J$. Atmos. Sci., 9, 493-505, 1974.

Woodman, R. F., and P. K. Rastogi, Evaluation of effective eddy diffusive coefficients using radar observations of turbulence in the stratosphere, Geophys. Res. Lett., 11, 243-246, 1984.

Yoe, J. G.,P. Czechowsky, R. Rüster, and G. Schmidt, Spatial variability of the aspect sensitivity of VHF radar echoes in the troposphere and lower stratosphere during jet stream passages, Ann. Geophys., 12, 733-745, 1994.

M. Crochet and H. Luce, LSEET, Université de Toulon et du Var, CNRS UA 705, B.P. 132, 83957, La Garde, France. (e-mail: luce@Iseet.univ-tln.fr)

F.Dalaudier and C. Sidi, Service d'Aéronomie du CNRS, Verrières le Buisson, France (e-mail: dalaudie@savtcp.aerov.jussieu.fr)

(Received December 4, 1995; revised May 14, 1996; accepted July 10, 1996) 